\title{
Complex Molecular Relationship Between Vegetative Compatibility Groups (VCGs) in Verticillium dahliae: VCGs Do Not Always Align with Clonal Lineages
}

\author{
María del Mar Jiménez-Gasco, Glenna M. Malcolm, Mónica Berbegal, Josep Armengol, and Rafael M. Jiménez-Díaz
}

First and second authors: Department of Plant Pathology and Environmental Microbiology, The Pennsylvania State University, University Park 16802; third and fourth authors: Instituto Agroforestal Mediterráneo, Universidad Politécnica de Valencia, Camino de Vera s/n, 46022 Valencia, Spain; and fifth author: College of Agriculture and Forestry, Universidad de Córdoba, Campus de Excelencia Internacional Agroalimentario, ceiA3, and Instituto de Agricultura Sostenible, CSIC, Alameda del Obispo s/n, P.O. Box 4084, 14080 Córdoba, Spain.

Current address of G. M. Malcolm: Department of Plant Science, The Pennsylvania State University, University Park 16802.

Accepted for publication 25 November 2013.

\begin{abstract}
Jiménez-Gasco, M. M., Malcolm, G. M., Berbegal, M., Armengol, J., and Jiménez-Díaz, R. M. 2014. Complex molecular relationship between vegetative compatibility groups (VCGs) in Verticillium dahliae: VCGs do not always align with clonal lineages. Phytopathology 104:650-659.

Verticillium wilts caused by the soilborne fungus Verticillium dahliae are among the most challenging diseases to control. Populations of this pathogen have been traditionally studied by means of vegetative compatibility groups (VCGs) under the assumption that VCGs comprise genetically related isolates that correlate with clonal lineages. We aimed to resolve the phylogenetic relationships among VCGs and their subgroups based on sequences of the intergenic spacer region (IGS) of the ribosomal DNA and six anonymous polymorphic sequences containing singlenucleotide polymorphisms (VdSNPs). A collection of $68 \mathrm{~V}$. dahliae isolates representing the main VCGs and subgroups (VCGs 1A, 1B, 2A, 2B, $3,4 \mathrm{~A}, 4 \mathrm{~B}$, and 6) from different geographic origins and hosts was analyzed using the seven DNA regions. Maximum parsimony (MP) phyloge-

nies inferred from IGS and VdSNP sequences showed five and six distinct clades, respectively. Phylogenetic analyses of individual and combined data sets indicated that certain VCG subgroups (e.g., VCGs 1A and 1B) are closely related and share a common ancestor; however, other subgroups (e.g., VCG 4B) are more closely related to members of a different VCG (e.g., VCG 2A) than to subgroups of the same VCG (VCG 4B). Furthermore, MP analyses indicated that VCG 2B is polyphyletic, with isolates placed in at least three distinct phylogenetic lineages based on IGS sequences and two lineages based on VdSNP sequences. Results from our study suggest the existence of main VCG lineages that contain VCGs $1 \mathrm{~A}$ and 1B; VCGs $2 \mathrm{~A}$ and $4 \mathrm{~B}$; and VCG 4A, for which both phylogenies agree; and the existence of other VCGs or VCG subgroups that seem to be genetically heterogeneous or show discrepancies in their phylogenetic placement: VCG 2B, VCG 3, and VCG 6. These results raise important caveats regarding the interpretation of VCG analyses: genetic homogeneity and close evolutionary relationship between members of a VCG should not be assumed.
\end{abstract}

Vegetative compatibility in fungi refers to the ability of hyphae to anastomose and form a stable heterokaryon. This allows organizing isolates that are vegetatively compatible into vegetative compatibility groups (VCGs). Based on knowledge derived from model fungal systems, vegetative compatibility is controlled by heterokaryon incompatibility (het) or vegetative incompatibility (vic) loci (21,31). For hyphal anastomosis to occur between two fungal isolates, alleles at those het or vic loci must be identical, or compartmentalization and hyphal cell death will occur at the point of fusion $(20,21,31)$. The apparent need of allele homozygosis at het or vic loci has led to the assumption that isolates within a VCG must be genetically related; consequently, VCGs are generally conceived as clonal lineages that comprise individuals related by common descent, with variation within clonal lineages arising mainly through mutations $(1,19)$.

The VCG structure in fungal populations has been used to estimate genetic diversity in many fungi $(38,47)$, including Verti-

Corresponding author: M. M. Jiménez-Gasco;

E-mail address: jimenez-gasco@psu.edu

* The $\boldsymbol{e}$-Xtra logo stands for "electronic extra" and indicates that the online version contains one supplemental table and one supplemental figure.

http://dx.doi.org/10.1094/PHYTO-07-13-0180-R

(C) 2014 The American Phytopathological Society cillium dahliae, a soilborne anamorphic fungus in the phylum Ascomycota that causes vascular wilt diseases in hundreds of important crops worldwide $(10,22,29,42,48)$. Puhalla first identified VCGs in $V$. dahliae using UV-induced microsclerotial color mutants (44). Based on this assay, four VCGs were initially identified (44), although this number increased to 16 VCGs when a diverse collection of 86 isolates was analyzed (45). Later, Joaquim and Rowe (26) reclassified 15 of the 16 VCGs identified by Puhalla (44) into four main VCGs (VCG 1, VCG 2, VCG 3, and VCG 4) using an assay that relies on the use of nitratenonutilizing (nit) auxotrophic mutants that arise naturally in medium containing chlorate (26). The use of nitrogen sources and the wild-type growth are reestablished following positive complementation between different nit mutants of paired isolates, and those isolates are assigned to the same VCG. Since 1990, VCG groups based on complementation assays with nit mutants have been slightly updated. Several VCGs have been further subdivided into subgroups based on vigor and frequency of complementation (i.e., VCG $1 \mathrm{~A}$ and VCG $1 \mathrm{~B}$, VCG $2 \mathrm{~A}$ and VCG 2B, and VCG 4A and VCG 4B) $(6,23,26,51)$. More recently, isolates causing disease on pepper in California were characterized as a new VCG 6 (9).

The advancement of molecular tools and their increased availability in phytopathology has led to a steady replacement of VCG testing by molecular assays and protocols to resolve fungal population diversity. However, VCGs are still important markers 
for studies that focus on $V$. dahliae $(23,41)$. Different VCGs can be linked to host adaptation shown by populations of $V$. dahliae and, therefore, these groups bear significant biological information $(8,23,24,28,48)$. In some cases, virulence to a given symptomatic host plant (34) can be correlated to different VCGs. For example, isolates of VCG 4A, which has only been found in North America, mainly associated with potato crops $(41,48)$, are more virulent on potato than VCG $4 \mathrm{~B}$ and $2 \mathrm{~B}$ isolates, which occur in North America and elsewhere (27,51). In Israel, VCG 4B isolates were more virulent on potato and VCG $2 \mathrm{~A}$ isolates were more virulent on tomato than VCG $2 \mathrm{~B}$ isolates (55). In Spain, V. dahliae isolates from artichoke in VCG $2 \mathrm{~B}$ were more virulent on this host plant compared with isolates in VCG $1 \mathrm{~A}$ and VCG $2 \mathrm{~A}$ (23). Another example regarding the phytopathogenic significance of VCGs concerns $V$. dahliae causing disease on cotton and olive in Spain. Isolates in VCG 1A correlate to the highly virulent defoliating pathotype in olive and cotton crops, whereas isolates of VCGs $2 \mathrm{~A}, 2 \mathrm{~B}$, or 4B cause the lesser virulent, nondefoliating syndrome in these two symptomatic hosts $(22,24)$. Those differences in virulence on cotton and olive between defoliating and nondefoliating isolates have been shown consistently $(4,5$, 22,32,37).

Previous work utilizing a suite of molecular tools suggests that VCGs in V. dahliae may not represent distinct clonal lineages and may be more diverse than previously expected $(12,13,23)$. For instance, studies using amplified fragment length polymorphism (AFLP) markers revealed that VCG subgroups are not necessarily closely related $(12,13)$. This finding was supported by analyses of mitochondrial sequences (35). Similarly, studies on Verticillium wilt in artichoke crops in eastern-central Spain indicated that $V$. dahliae isolates from affected plants and typed as VCG 2B were genetically diverse because some isolates complemented with international reference VCG 2B tester isolates but others did not complement with these testers but did with local VCG 2B isolates (23). Polymerase chain reaction (PCR)-based markers previously associated with cotton- and olive-defoliating or nondefoliating $V$. dahliae pathotypes, as well as AFLP analysis, also revealed this genetic heterogeneity $(14,23,37)$.

The objective of this study was to analyze phylogenetic relationships between VCGs in V. dahliae to better understand the population structure in this important pathogen. This has been attempted before using AFLPs $(12,13)$, conserved, housekeeping genes (12), and mitochondrial DNA regions (35). However, results from those studies may be undermined by AFLP markers being potentially subjected to significant homoplasy (17), and by the little resolution within VCGs provided by other DNA regions tested $(12,35)$. Also, not all known VCGs were included in previous studies $(12,35)$. A region that has shown promise in $V$. dahliae is the nuclear ribosomal DNA (rDNA) gene cluster intergenic spacer (IGS). Qin et al. (46) showed significant variability in IGS sequences in $V$. dahliae populations from numerous symptomatic hosts but VCG information was not included in the analyses. Other sequence-based regions of potential use for studying VCG phylogenetic relationships are those containing single-nucleotide polymorphisms (SNPs) $(15,54)$. In the present study, we analyzed evolutionary relationships between VCGs using IGS sequences and six previously characterized anonymous polymorphic regions containing SNPs (7). We hypothesized that clonality within VCGs of $V$. dahliae will be supported by topological concordance between the different regions analyzed; that is, if VCGs comprise clonal lineages, the topologies of phylogenetic trees inferred from the different data sets (IGS and the six polymorphic regions) should be congruent in the absence of recombination.

\section{MATERIALS AND METHODS}

Fungal isolates, culture conditions, and DNA extraction. A collection of 68 isolates of $V$. dahliae and one of $V$. albo-atrum originally obtained from diverse hosts, geographic origins, and VCG groups were used in this study (Table 1). The collection included isolates from China, Greece, Israel, Italy, Japan, Spain, Swaziland, Syria, the United Kingdom, and the United States. The host of origin of the isolates was also diverse, including artichoke, chickpea, cotton, eggplant, elm, green ash, muskmelon, olive, pepper, pistachio, potato, pumpkin, strawberry, tomato, watermelon, and yellowwood (Table 1). All V. dahliae isolates had been previously typed to VCG in earlier studies or by the supplier (Table 1). The collection studied includes all known VGCs and subgroups described, except for VCG 5. VCG 5 was only described once for a single isolate from catalpa in Illinois (United States) $(26,45,52)$, which is no longer available. Isolates are long-term stored in sterile soil at $4{ }^{\circ} \mathrm{C}$ in the dark, in the culture collection of M. M. Jiménez-Gasco, Department of Plant Pathology and Environmental Microbiology, The Pennsylvania State University. Active cultures were obtained by plating colonized soil onto potato dextrose agar (PDA; Difco Laboratories, Detroit), followed by incubation at $26^{\circ} \mathrm{C}$ in the dark. Efforts were made to assure purity of isolates' cultures. Mycelium was generated from 2- to 4-week-old PDA cultures by gently scraping it off the media. This mycelium was used directly for DNA extraction using the Mo Bio Ultraclean Microbial DNA Isolation kit (Mo Bio Laboratories Inc., Carlsbad, CA) following the manufacturer's protocol, with the addition of $10 \mathrm{~min}$ at $65^{\circ} \mathrm{C}$ after step 4 to increase DNA yield through improved cell lysis. DNA solutions were stored at $-20^{\circ} \mathrm{C}$ until use.

Amplification and sequencing of IGS and polymorphic regions containing SNPs. All 69 Verticillium isolates were subjected to phylogenetic analyses using IGS sequences and six polymorphic regions containing SNPs (VdSNP sequences) (7). Conditions used for DNA amplification of the complete IGS region were as described by Qin et al. (46) using primers VdIGSF1 and VdIGSR1 (46). For amplification of the IGS region, the reaction mix $(25 \mu \mathrm{l})$ consisted of $20 \mathrm{ng}$ of DNA, $0.5 \mu \mathrm{M}$ each primer, $0.2 \mathrm{mM} \mathrm{dNTP}, 2.5 \mu \mathrm{l}$ of $10 \times$ reaction buffer, and $0.65 \mathrm{U}$ of DNA polymerase (Denville Scientific, Metuchen, NJ). All PCR reactions were performed in a Mastercycle-ep Thermocycler (Eppendorf North America, Hauppauge, NY), with an initial denaturation step at $95^{\circ} \mathrm{C}$ for $5 \mathrm{~min}$; followed by 35 cycles of $1 \mathrm{~min}$ at $95^{\circ} \mathrm{C}, 50 \mathrm{~s}$ at $65^{\circ} \mathrm{C}$, and $1 \mathrm{~min}$ at $72^{\circ} \mathrm{C}$; and a final step of $10 \mathrm{~min}$ at $72^{\circ} \mathrm{C}$. All polymorphic regions containing SNPs (VdSNP1, 2, 3, 4, 5, and 7) were amplified using primers and protocols described by Berbegal et al. (7). Amplification products were visualized in agarose gels prior to sequencing. PCR products were cleaned using ExoSap (Affimetrix USB, Cleveland), and sequenced at the Genomics Core Facility, The Pennsylvania State University, University Park. Sequencing of the complete IGS region was achieved by using primers VdIGSF1 and VdIGSR1 (46), as well as primers VdIGSInt1F (5'-CTTTCGGCTGCA GCGGCGTGCC-3'), VdIGSInt1R (5'-GGCACGCCGCTGCA GCCGAAAG- $3^{\prime}$ ), and VdIGSInt2R (5'-AATTCCCGGGTAGCTT TCCACC-3'), which were designed in this study based on sequences internal to the IGS region. Both strands were sequenced for all VdSNP regions using the same primers used for PCR amplifications, and consensus sequences for IGS and VdSNP regions were generated using Sequencher (v.4.7; Gene Codes, Ann Arbor, MI).

DNA sequence analysis. For each region, sequences were aligned using Sequencher v.4.7 followed by careful and extensive manual alignment, especially for the IGS region. Manual alignment was advisable because the IGS region contains numerous large indels, as described by Pramateftaki et al. (43). Congruence between all data sets was estimated with the incongruence length difference (ILD) test implemented as the partition homogeneity test in PAUP v.4.0.b10 (53), with 1,000 replicate partitions subjected to heuristic parsimony searches. Phylogenetic analyses were conducted on each data set individually, all VdSNP se- 
quences combined, and on the combined data set. Maximum parsimony (MP) analyses were conducted using the parsimony ratchet (39) in PAUPRat (50) as implemented in PAUP v.4.0.b10, with the following settings: set seed $=0$, nreps $=200$, pct $=15$, set wtmode $=$ uniform, with simple sequence addition and heuristic searches. Maximum likelihood (ML) analyses were done in PAUP using the HKY85 model and the heuristic search option, with 1,000 random addition sequences and the subtree-punningregrafting algorithm. An isolate of $V$. albo-atrum was used as outgroup. MP and ML bootstrapping were conducted using 1,000 replicates. The Shimodaira-Hasewaga test (49) was used to evaluate the alternative phylogenetic hypothesis of monophyly for VCG 2B under ML. This test was implemented in PAUP with 1,000 replicates.

\section{RESULTS}

Amplification and sequencing of IGS and VdSNP regions. Amplification of the complete IGS region of the nuclear rDNA resulted in single amplicons of 1,700 to $2,200 \mathrm{bp}$ in size, depending on the isolate. After editing sequences and alignments, the IGS region was 1,743 to 2,328 bp for all Verticillium isolates tested, including gaps and indels. The length of the VdSNP sequences did not vary among isolates and coincided with the values described by Berbegal et al. (7). After editing of sequences and completion of alignments, the length for each polymorphic sequence data set, including gaps, was as follows: VdSNP1, 244 bp; VdSNP2, 411 bp; VdSNP3, 262 bp; VdSNP4, 298 bp;
VdSNP5, 417 bp; and VdSNP, 209 bp. All sequences generated in this study were submitted to the National Center for Biotechnology Information GenBank and have been deposited under accession numbers KF295831 to KF296313.

Phylogenetic analyses. Partition homogeneity tests between all pair wise combinations of data sets were conducted to assess concordance between the different regions (Supplemental Table 1). ILD tests showed that certain data sets were congruent but incongruence was statistically significantly for the following data set combinations: VdSNP1/VdSNP3, VdSNP1/VdSNP5, VdSNP1/ VdSNP7, IGS/VdSNP1, IGS/VdSNP5, IGS/VdSNP7, and VdSNP5/VdSNP7 $(P<0.05)$. Phylogenetic analyses were done on (i) the IGS data set alone, (ii) each of the VdSNP polymorphic regions individually, (iii) all the VdSNP regions combined, and (iv) all regions (IGS and VdSNP) combined. For all data sets, both MP and ML analyses were performed; however, because trees generated with both types of analyses were nearly identical (data not shown), only MP phylogenetic trees are shown (Figs. 1 and 2). MP analysis of the IGS region yielded five distinct clades (clades 1 to 5) (Fig. 1A). Clade 1 included exclusively all isolates of VCG 4A (74\% bootstrap support). Clade 2 grouped 7 of the 13 VCG 2B isolates included in the analysis (Fig. 1A, isolates 2B-I) (61\% bootstrap support). Clade 3 comprised all isolates typed as VCG 1 (including VCG $1 \mathrm{~A}$ and VCG 1B), as well as three VCG 2B isolates from artichoke in Spain (Fig. 1A, 2B-II) (85\% bootstrap support). Clade 4 was the most complex, including all isolates typed as VCG $2 \mathrm{~A}$ and VCG $4 \mathrm{~B}$, the three isolates of VCG 3 , and one isolate of VCG 2B (isolate CS1) (79\% bootstrap

TABLE 1. Isolates used in this study with reference numbers, geographic origin, host source, vegetative compatibility group (VCG), and referenced study where the VCG testing was reported

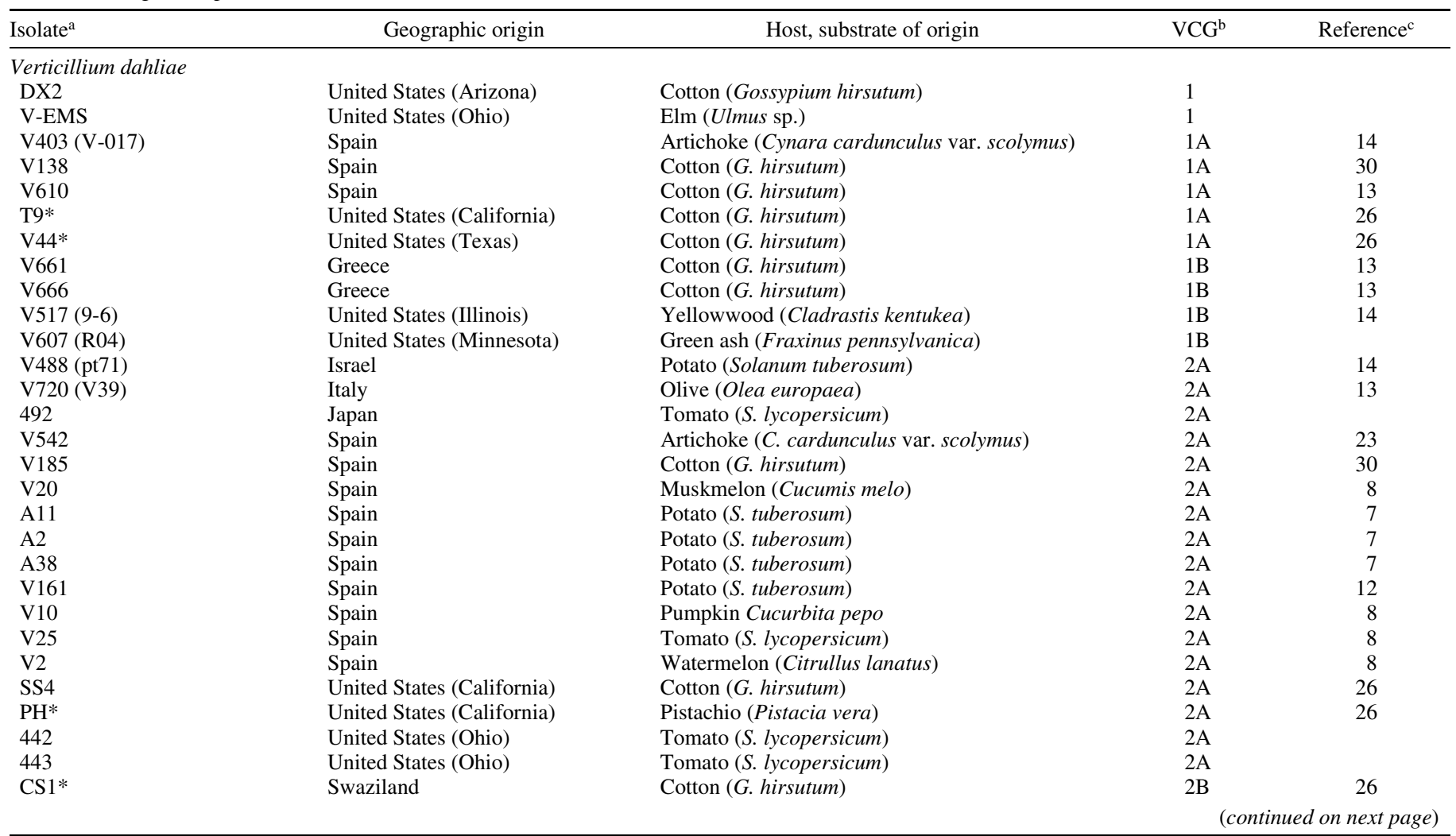

${ }^{a}$ Isolate reference. In parenthesis, codes given by supplier or by previous studies. An asterisk indicates that this isolate was used in the original VCG classification by Puhalla and Hummel (45). Isolates from Greece were provided by E. Paplomatas, Agricultural University of Athens, Athens; isolates from Israel and the United States (California) were provided by T. Katan, The Volcani Center, Bet Dagan, Israel; isolates from the United Kingdom, California, and Brazil were provided by D. Barbara, Warwick HRI, England; isolates from the United States and Japan were provided by R. Rowe, OARDC, The Ohio State University, Wooster; and isolates from Italy were provided by F. Nigro, Università degli Studi di Bari, Bari, Italy, or A. Zazzerini, Università degli Studi di Perugia, Perugia, Italy.

${ }^{\mathrm{b}}$ VCG was determined in the study referenced in the next column or by the supplier of the isolate. HSI $=$ heterokaryon self-incompatible and $\mathrm{n} / \mathrm{a}=$ not applicable

${ }^{\mathrm{c}}$ References of studies where isolates were assigned to VCG. For isolates with no reference, VCG typing was performed by the supplier.

d VCG 2Ba, artichoke isolate was able to complement with other artichoke isolates assigned to VCG2B but not with international VCG 2B testers. 
support). Finally, clade 5 comprised isolates of VCG 6 originating from pepper in California and three VCG $2 \mathrm{~B}$ isolates from artichoke in Spain (Fig. 1A, 2B-III) (69\% bootstrap support). There were two isolates included in the analyses that failed to self-anastomose and were typed as heterokayon self-incompatible (HSI). Those two isolates were placed in different clades in the IGS MP tree; one (V547) was placed within clade 3, closely related to VCGs $1 \mathrm{~A}$ and $1 \mathrm{~B}$, and the second one (PU) was placed within clade 5, together with VCG 6 and VCG 2B-III isolates (Fig. 1A).

Results of analyses of individual VdSNP polymorphic sequences are shown in Supplemental Figure 1. For individual regions, clade resolution was poor due to the limited number of polymorphic sites (2 to 10 parsimony-informative characters); therefore, all VdSNP regions were combined into one data set, even though some regions were incongruent. An MP tree of all six VdSNP polymorphic sequences combined is shown in Figure 1B and yielded six main clades (I to VI). Clade I included all isolates of VCG 4A (63\% bootstrap support) and was closely related to clade II that comprised the two isolates of VCG 6 analyzed (75\% bootstrap support). Clade III was closely related to clades I and II and included 8 of the 13 isolates of VCG $2 \mathrm{~B}$ and the HSI isolate PU (bootstrap support of 85\%). Clade IV comprised all isolates of VCGs $2 \mathrm{~A}$ and 4B (70\% bootstrap). Clade V contained all isolates of VCGs 1A, 1B, and 3 (68\% bootstrap). A final clade, VI, included three isolates of VCG 2B and the HSI isolate V547 (72\% bootstrap). Two isolates from VCG 2B (isolates 115 and V652) remained unresolved (Fig. 1B).
With the exception of VCG $2 \mathrm{~B}$, isolates within a VCG subgroup were closely related to each other. For most VCGs (i.e., VCGs 4A, 2A, 4B, 1A, and 1B), the IGS and VdSNP phylogenies were congruent. For these phylogenies, isolates within a VCG subgroup were, indeed, closely related to each other, sharing a common ancestor. For example, all isolates of VCG 4A were placed in the same clade for both phylogenies (clades 1 and I for IGS and VdSNP, respectively) (Fig. 1). Regarding relationships between subgroups of a VCG, results showed that VCGs $1 \mathrm{~A}$ and $1 \mathrm{~B}$ are closely related and isolates from both subgroups are indistinguishable based on either phylogeny. However, this was not the case for other VCG subgroups. VCGs 4A and 4B were not phylogenetically related, and isolates of VCG 4B were indistinguishable phylogenetically from isolates of VCG 2A. For other groups (i.e., VCGs 3, 6, and 2B), the IGS and VdSNP phylogenies were not concordant and these isolates were placed in different clades. VCG 3 isolates were nested within clade 4 based on IGS, and indistinguishable from isolates of VCGs $2 \mathrm{~A}$ and 4B (Fig. 1A). However, isolates of VCG 3 were grouped together with isolates of VCG $1 \mathrm{~A}$ and $1 \mathrm{~B}$ in clade $\mathrm{V}$ based on VdSNP sequences (Fig. 1B). A similar situation occurred for VCG 6, which grouped with VCG 2B-III isolates in IGS clade 5 (Fig. 1A), but they were placed in clade II, closely related to VCG 4A (clade I), based on VdSNP sequences (Fig. 1B). IGS and VdSNP phylogenies showed the most complex relationships for isolates of VCG 2B. These were divided into three groups (named 2B-I, 2B-II, and 2B-III) in the IGS phylogeny (clades 2, 3, and 5),

TABLE 1. (continued from preceding page)

\begin{tabular}{|c|c|c|c|c|}
\hline Isolate $^{\mathrm{a}}$ & Geographic origin & Host, substrate of origin & $\mathrm{VCG}^{\mathrm{b}}$ & Reference $^{c}$ \\
\hline V357 (JY) & China & Cotton (G. hirsutum) & 2B & 13 \\
\hline V652 & Greece & Cotton (G. hirsutum) & $2 \mathrm{~B}$ & 13 \\
\hline $\mathrm{V} 285(\cot 72)$ & Israel & Cotton (G. hirsutum) & 2B & 30 \\
\hline V258 (CECE) & Italy & Chickpea (Cicer arietinum) & 2B & 12 \\
\hline V534 & Spain & Artichoke (Cynara cardunculus var. scolymus) & $2 \mathrm{~B}$ & 23 \\
\hline V549 & Spain & Artichoke (C. cardunculus var. scolymus) & $2 \mathrm{~B}$ & 23 \\
\hline V574 & Spain & Artichoke (C. cardunculus var. scolymus) & $2 \mathrm{~B}^{\mathrm{d}}$ & 23 \\
\hline V593 & Spain & Artichoke (C. cardunculus var. scolymus) & $2 \mathrm{~B}$ & 23 \\
\hline V613 & Spain & Artichoke (C. cardunculus var. scolymus) & 2B & 23 \\
\hline $115^{*}$ & Syria & Cotton $(G$. hirsutum $)$ & $2 \mathrm{~B}$ & 26 \\
\hline V552 (332) & United Kingdom & Strawberry $($ Fragaria $\times$ ananassa $)$ & $2 \mathrm{~B}$ & 12 \\
\hline P19 & United States (Ohio) & Potato $(S$. tuberosum $)$ & $2 \mathrm{~B}$ & 26 \\
\hline S92 & United States (Ohio) & Potato soil & $2 \mathrm{~B}$ & 26 \\
\hline 70.21 & United States (Arizona) & Bell pepper (Capsicum апnиит) & 3 & 26 \\
\hline $\mathrm{PCW}^{*}$ & United States (California) & Pepper $($ C. аппиит $)$ & 3 & 26 \\
\hline VMD9 & United States (Oregon) & Peppermint $($ Mentha $\times$ piperita $)$ & 3 & \\
\hline V25R & United States (Idaho) & Potato (S. tuberosum) & $4 \mathrm{~A}$ & \\
\hline V27 & United States (Idaho) & Potato (S. tuberosum) & $4 \mathrm{~A}$ & \\
\hline 318 & United States (North Dakota) & Potato (S. tuberosum) & $4 \mathrm{~A}$ & 27 \\
\hline 319 & United States (North Dakota) & Potato (S. tuberosum) & $4 \mathrm{~A}$ & 27 \\
\hline 320 & United States (North Dakota) & Potato (S. tuberosum) & $4 \mathrm{~A}$ & 27 \\
\hline $321 d$ & United States (North Dakota) & Potato (S. tuberosum) & $4 \mathrm{~A}$ & \\
\hline VA102 & United States (North Dakota) & Potato (S. tuberosum) & $4 \mathrm{~A}$ & \\
\hline VA49 & United States (North Dakota) & Potato (S. tuberosum) & $4 \mathrm{~A}$ & \\
\hline V304 (cot 120) & Israel & Cotton $(G$. hirsutum $)$ & 4B & 30 \\
\hline V683 & Spain & Artichoke (Cynara cardunculus var. scolymus) & 4B & 23 \\
\hline V684 & Spain & Artichoke (C. cardunculus var. scolymus) & $4 \mathrm{~B}$ & 23 \\
\hline V158 & Spain & Eggplant (S. melongena) & 4B & 8 \\
\hline V789 & Spain & Olive $($ O. europaea $)$ & $4 \mathrm{~B}$ & 13 \\
\hline A54 & Spain & Potato (S. tuberosum) & $4 \mathrm{~B}$ & 7 \\
\hline V61 & Spain & Potato $(S$. tuberosum $)$ & $4 \mathrm{~B}$ & 7 \\
\hline V551 (330) & United Kingdom & Strawberry $($ Fragaria $\times$ ananassa $)$ & $4 \mathrm{~B}$ & 12 \\
\hline V553 (1875) & United Kingdom & Strawberry (Fragaria $\times$ ananassa) & $4 \mathrm{~B}$ & 12 \\
\hline S39 & United States (Ohio) & Potato soil & $4 \mathrm{~B}$ & 26 \\
\hline WS4 & United States (Wisconsin) & Potato (S. tuberosum) & $4 \mathrm{~B}$ & \\
\hline V560 (VdCa.83a) & United States (California) & Pepper (Capsicum annuиm) & 6 & 9 \\
\hline V561 (VdCa147a) & United States (California) & Pepper (C. аппиит) & 6 & 9 \\
\hline V547 & Spain & Artichoke (Cynara cardunculus var. scolymus) & HSI & 23 \\
\hline PU* & United Kingdom & Potato (S. tuberosum) & HSI & 26 \\
\hline \multicolumn{5}{|l|}{ V. albo-atrum } \\
\hline 462 & United States (Minnesota) & Potato (S. tuberosum) & $\mathrm{n} / \mathrm{a}$ & \\
\hline
\end{tabular}


and into two groups in the VdSNP one (in clades III and VI) (Figs. 1 and 3). Isolates of VCG 2B-I, placed in clade 2 based on IGS, were included in VdSNP clade III together with isolates of VCG 2B-III (IGS clade 5). All isolates of 2B-II were indistinguishable from VCG $1 \mathrm{~A}$ and $1 \mathrm{~B}$ isolates and placed in IGS clade 3. However, 2B-II isolates were grouped in VdSNP clade VI, distinct from VCGs $1 \mathrm{~A}$ and 1B (Fig. 1). Also, isolate 2B-I V652 was closely related to VdSNP clade VI. A comparison of both IGS and VdSNP phylogenies is shown in Figure 3, indicating the lack of congruence in the placement of VCGs $2 \mathrm{~B}, 3$, and 6 in both phylogenies.

Finally, an MP tree was inferred from the combined data set including IGS and the six VdSNP polymorphic sequences (Fig. 2 ), although IGS was not congruent with the combined VdSNP data set $(P<0.001)$. Overall, the MP tree inferred from the combined data (Fig. 2) was very similar to the IGS tree (Fig. 1A), which was not unexpected because the IGS region contributed 94 parsimony-informative characters to the analysis, whereas the combined VdSNP data set contributed only 30 parsimony-informative characters of a total of 123. Three main, well-supported clades were resolved in the combined phylogenetic tree: one comprised all VCG 4A isolates (97\% bootstrap support); a second included all isolates of VCGs 1A, 1B, and some isolates of VCG 2B (termed earlier VCG 2B-II isolates) (93\% bootstrap support); and a third clade included isolates of VCGs 2A, 2B-III, 3, 4B, and 6 (79\% bootstrap support). The rest of VCG $2 \mathrm{~B}$ isolates (called earlier VCG 2B-I) remained unresolved in a poorly supported clade closely related to VCG $4 \mathrm{~A}$. One important observation is that bootstrap values were higher in the combined analysis (Fig. 2) compared with the IGS tree (Fig. 1A) or the combined VdSNP tree (Fig. 1B), but only for those clades with a concordant phylogenetic placement in both individual phylogenies (i.e., VCG 4A or VCGs $1 \mathrm{~A}$ and $1 \mathrm{~B}$ ). However, bootstrap values dropped for the analysis of combined data sets in the rest of the clades (Figs. 1 and 2). As indicated by the IGS and VdSNP trees, isolates of VCG 2B were present in different well-supported clades of the combined data set, indicating polyphyly of this group. The alternative hypothesis of monophyly for VCG $2 \mathrm{~B}$ was tested using the Shimodaira-Hasewaga test by comparing a tree in which isolates of VCG 2B were constrained to be monophyletic with the unconstrained tree. The analysis showed that the constrained tree was significantly less likely ( $\ln L=-8,821.399$ ) than the uncon-
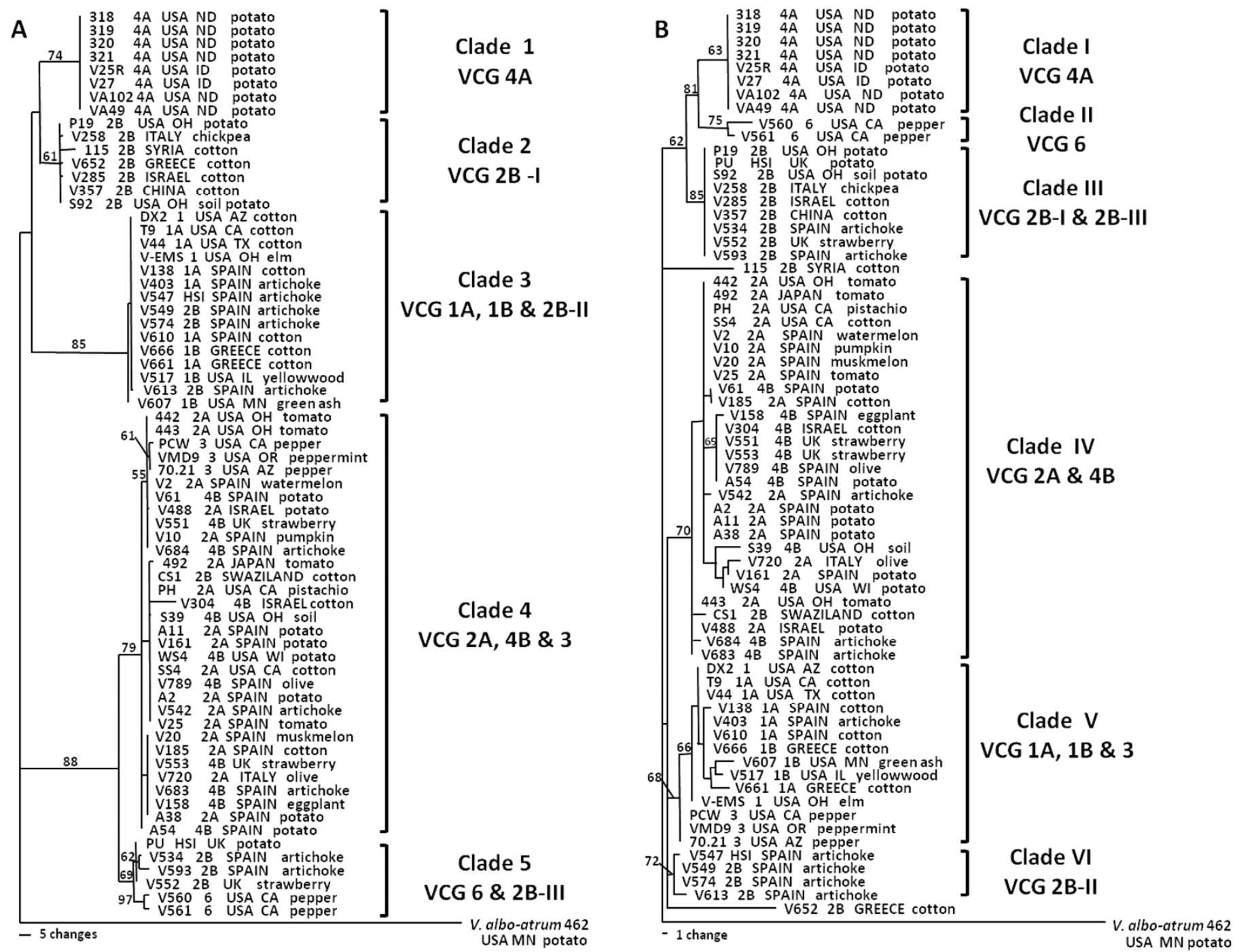

Fig. 1. Phylogenetic relationships between Verticillium dahliae vegetative compatibility groups (VCGs). A, One of 200 most parsimonious phylogenetic trees inferred from sequences of the intergenic spacer (IGS) region of the ribosomal DNA ( 93 parsimony-informative characters, 327 steps, consistency index $[\mathrm{CI}]=$ 0.930 , retention index $[R I]=0.966$, rescaled consistency index $[R C]=0.898)$. B, One of 200 most parsimonious phylogenetic trees inferred from combined polymorphic sequences containing single-nucleotide polymorphisms (30 parsimony-informative characters, 163 steps, $\mathrm{CI}=0.791, \mathrm{RI}=0.901, \mathrm{RC}=0.713$ ) Numbers above branches are bootstrap values $(>50 \%)$ based on 1,000 replicates. Each taxon label indicates isolate reference, VCG, geographical origin, and host source. Trees are rooted with V. albo-atrum 462. VCG 2B isolates are further subdivided into VCG 2B-I, VCG 2B-II, and VCG 2B-III according to their phylogenetic placement in the IGS tree (A). 
strained tree $(\ln L=-9,339.372)(P=0.000)$, strongly supporting polyphyly for VCG $2 \mathrm{~B}$.

\section{DISCUSSION}

For the last two decades, VCG analysis has been used to estimate genetic variation in $V$. dahliae populations $(6,9,16,18$, 23,26-28,30,51), under the assumption that isolates within a given VCG comprise genetically related isolates that descend from a common ancestor; that is, VCGs align with clonal lineages. This has been shown to be true for many fungi $(3,25,36,47)$. However, results from this and other studies indicate that, for some VCGs in V. dahliae, this assumption is not necessarily true, and a VCG may comprise a genetically heterogeneous group of isolates that are phylogenetically distant, as shown herein for VCG $2 \mathrm{~B}$ based on IGS and VdSNP analyses.

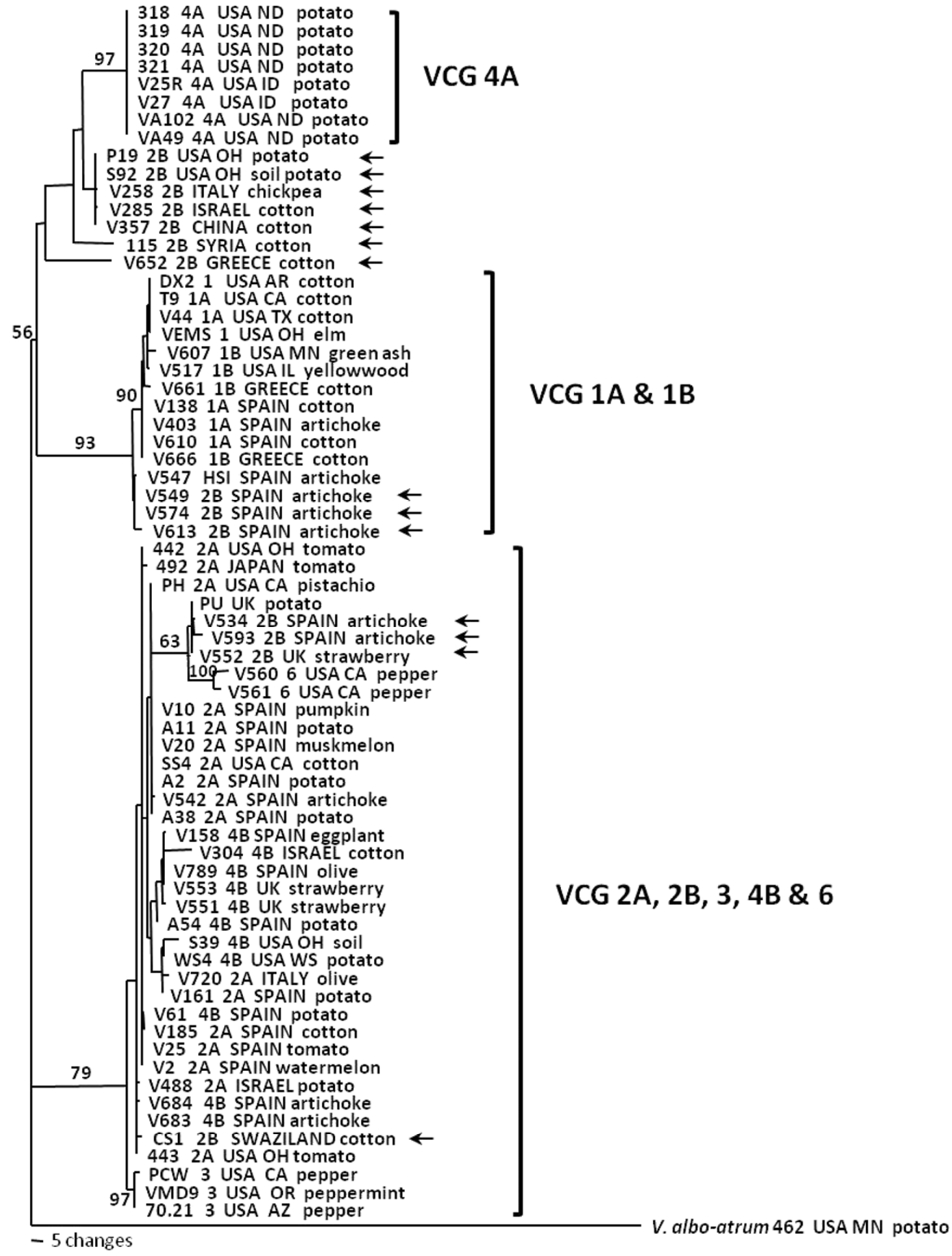

Fig. 2. Phylogenetic relationships between Verticillium dahliae vegetative compatibility groups (VCGs). One of 200 most parsimonious phylogenetic trees inferred from the combined dataset of the intergenic spacer region of the rDNA and six polymorphic sequences containing single-nucleotide polymorphisms (123 parsimony-informative characters, 504 steps, consistency index $=0.859$, retention index $=0.931$, rescaled consistency index $=0.8$ ). Numbers above branches are bootstrap values $(>50 \%)$ based on 1,000 replicates. Each taxon label indicates isolate reference, VCG, geographical origin, and host source. Trees are rooted with V. albo-atrum 462. Arrows indicate VCG 2B isolates. 
Another clear indication from the present study is that, with the exception of VCGs $1 \mathrm{~A}$ and $1 \mathrm{~B}$ that are closely related, all other subgroups for a given VCG are not phylogenetically related, do not share a common ancestor, and, indeed, are closer to subgroups of other VCGs than to each other. For example, VCG 4A isolates are distinct from VCG 4B isolates, and VCG 4B isolates are phylogenetically indistinguishable from VCG $2 \mathrm{~A}$ isolates, regardless of the genomic region analyzed (Figs. 1 and 2). This is consistent with analyses based on mitochondrial sequences (34) but not with results from AFLP analyses that clearly distinguished between VCG 2A and VCG 4B (12). Isolates of VCG 2A and 4B have been shown to establish weak complementation, and some of these isolates have been said to be "bridge isolates" $(6,28)$. Whether this is an artifact of the nit-mutant VCG assay itself (6) or some "transitory" heterokaryosis (27) is unknown, and more research targeting het or vic genes and mechanisms of incompatibility in $V$. dahliae is needed.

We had hypothesized that clonality within VCGs of $V$. dahliae would be supported by topological concordance between the different regions analyzed. Although, for several VCGs, the phylogenies for IGS and VdSNP were congruent, this was not the case for all groups because there was significant disagreement in their phylogenetic placement. For example, VCGs 4A, 2A, 4B, $1 \mathrm{~A}$, and $1 \mathrm{~B}$ were grouped similarly based on IGS and VdSNP sequences (Fig. 2). However, VCG 3 isolates were nested within the clade containing isolates of VCGs $2 \mathrm{~A}$ and $4 \mathrm{~B}$ based on IGS (Fig. 1A, clade 4), whereas VCG 3 was closely related to VCG $1 \mathrm{~A}$ and $1 \mathrm{~B}$ according to the VdSNP phylogeny (Fig. 1B, clade V). Similarly, VCG 6 isolates grouped with some VCG 2B isolates in IGS clade 5 but were closely related to VCG $4 \mathrm{~A}$ according to VdSNP sequences (Fig. 1B). The most striking differences in both phylogenies involved VCG $2 \mathrm{~B}$ isolates. Whereas isolates of VCG 2B were placed in three different clades based on IGS, there were only two VdSNP clades containing VCG 2B isolates.
Isolates of 2B-I were placed in IGS clade 2 and within clade III in the VdSNP tree. This VdSNP clade also contained 2B-III isolates, placed in a separate distant clade (clade 5) in the IGS phylogeny. All 2B-II isolates were in clade VI (Fig. 2). Isolates named 2-II in this study contained isolate V574, characterized as VCG 2Ba by Jiménez-Díaz et al. (23). VCG 2Ba isolates were obtained from artichoke in eastern-central Spain and were unable to complement with international VCG $2 \mathrm{~B}$ testers but produced positive complementation with local VCG $2 \mathrm{~B}$ testers. Thus, those VCG $2 \mathrm{Ba}$ isolates were genetically different from other VCG $2 \mathrm{~B}$ isolatescalled VCG $2 \mathrm{Br}$ by Jiménez-Díaz et al. (23) — that did complement with international reference VCG $2 \mathrm{~B}$ testers $(23,30)$. There was also diversity in PCR-based amplicons of 334 and $824 \mathrm{bp}$, markers previously associated with cotton- and olive-defoliating and nondefoliating $V$. dahliae pathotypes, respectively $(14,23,37)$. Some VCG 2B isolates from artichoke amplified the defoliatingassociated marker of $334 \mathrm{bp}$, whereas others amplified the nondefoliating marker of $824 \mathrm{bp}(7,23)$. All 2B-II isolates, including the HSI isolate V547, grouped in IGS clade 3 and VdSNP clade VI, amplified the 334-bp marker, which is consistent with these isolates being closely related to cotton- and olive-defoliating VCG $1 \mathrm{~A}$ isolates. All other VCG $2 \mathrm{~B}$ isolates analyzed amplified the 824-bp marker $(7,12,23)$. An earlier study that included some of the VCG 2B isolates that amplified the 824-bp marker showed identical haplotypes for five microsatellites (7), which was different from the haplotypes displayed by VCG 2B isolates that amplified the 334-bp marker. The discrepancies between phylogenies generated by IGS and VdSNP sequences could also be explained by the fast-evolving nature of the IGS region. Similar incongruencies between IGS and other regions have been reported for the Fusarium oxysporum complex $(19,40)$.

Overall, comparisons of results from the present study with those from AFLP analysis (12) indicate that some of the groups generated with AFLP phylogenies coincide with the VCG rela-

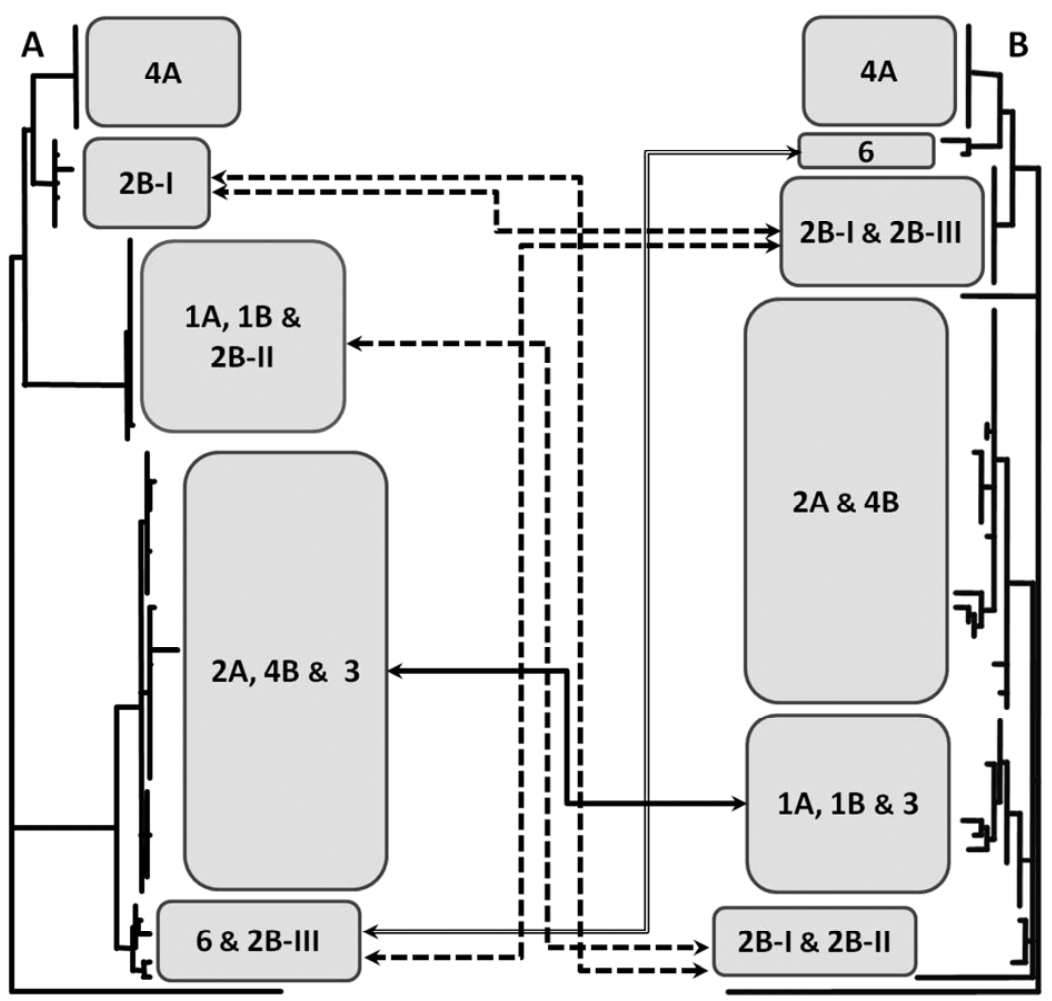

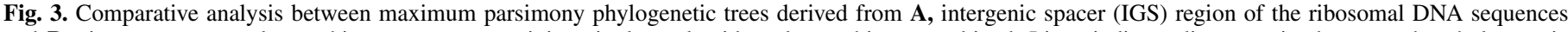

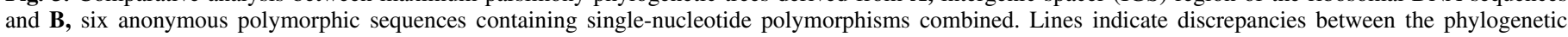

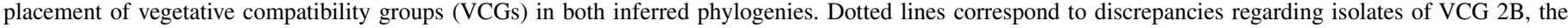

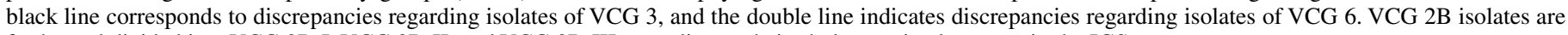
further subdivided into VCG 2B-I, VCG 2B-II, and VCG 2B-III according to their phylogenetic placement in the IGS tree. 
tionships determined by the IGS phylogeny but some others are closer to the clades displayed by the VdSNP phylogeny. Because AFLP markers are widespread throughout the whole genome, it would be logical to expect that the AFLP tree would look like a combination of the phylogenies inferred from IGS and VdSNP polymorphic regions in the present study. Although ColladoRomero et al. (12) included a sequence analysis of various other regions (housekeeping genes actin, $\beta$-tubulin, calmodulin, and histone 3, as well as the ITS2 region of the rDNA), these regions resolved very little diversity, and most of the phylogenetic signal in the analysis combining AFLPs and other regions was contributed by the AFLP markers. Thus, the AFLP study (12) identified two main evolutionary groups: lineage I that corresponds to IGS clade 3 and lineage II that includes the rest of IGS clades identified in the present research. In the AFLP dendrogram, VCG 6 isolates grouped with the only isolate of VCG 4A included in that study, which coincides with the close placement of these two VCGs using VdSNP sequences (Fig. 1B).

Our results indicate that VCG $2 \mathrm{~B}$ is clearly polyphyletic, which was also noted by Collado-Romero et al. (12) using AFLP markers, although only two VCG $2 \mathrm{~B}$ groups were identified by that study. Dung et al. (18) also identified two genetically distinct groups within VCG 2B isolates using microsatellite analysis: one group contained isolates from tomato and pistachio and the other comprised isolates from potato, watermelon, and mint. There are several possible explanations for polyphyly in VCGs. One is that convergent mutations in het or vic loci may have resulted in the same functional alleles that allow anastomosis and the establishment of stable heterokaryons between isolates of otherwise distinct genetic background. A second potential scenario is the exchange of genetic material between different VCGs due to parasexuality, especially during transitory heterokaryosis between different VCGs, as has been shown to be possible experimentally in Verticillium (56) and other fungi (33). In the latter case, the resulting genotypes would share genomic regions with both parental isolates. Finally, these isolates of conflicting phylogenetic placement may represent remnants of ancient, or current but rare, recombination events. $V$. dahliae populations have been shown to harbor MAT1-1 and MAT1-2 idiomorphs similar to the genetic mating system of other species of Ascomycota (2,57). Also, a departure from clonality shown by microsatellite analysis of $V$. dahliae populations was attributed to potential sexual recombination (2), although a major predominance of MAT1-2 was found in populations of $V$. dahliae, with only 1 isolate carrying the MAT1-1 idiomorph in 286 isolates tested (18). In any case, and regardless of the evolutionary mechanism that resulted in polyphyly of VCGs, results from this and other studies advise for an important caveat regarding the interpretation of VCG analyses: genetic homogeneity or close evolutionary relationships between members of a VCG should not be assumed.

In our study, we included some of Puhalla's original isolates $(44,45)$ that were used to perform early VCG testing $(6,27$, $45,51,52)$. One of those isolates, isolate PCW, was first typed as VCG 16 by Puhalla and Hummel (45), later reclassified as VCG 3 by Joaquim and Rowe (26), and finally identified as VCG 4 by Bell (6) and Strausbaugh et al. (52). Interestingly, in our study. this PCW isolate as well as others typed as VCG 3 sensu Joaquim and Rowe (26) were placed with VCGs $2 \mathrm{~A}$ and $4 \mathrm{~B}$ within IGS clade 4 and together with VCG $1 \mathrm{~A}$ and $1 \mathrm{~B}$ in clade $\mathrm{V}$ based on VdSNP sequences. VCG 3 was reported as being an interspecific hybrid between $V$. dahliae VCG1B and an unidentified non-Verticillium parent (11). This was due to the presence of two different sequences for actin, calmodulin, and histone 3 genes in the VCG tester isolate 70.21; one sequence that matched other $V$. dahliae sequences and another sequence that matched an unidentified non-Verticillium member of the phylum Ascomycota (11). Isolate 70.21 was also included in our analyses together with two other VCG 3 isolates. However, in contrast with
Collado-Romero et al. (11), in our study, all sequences associated with the three VCG 3 isolates were clean and there was no indication of multiple sequences contained in one amplicon. Based on our analysis, there is no indication of a hybrid origin for VCG 3 .

Our study suggests the existence of main VCG clonal lineages that contain the isolates of VCGs $1 \mathrm{~A}$ and $1 \mathrm{~B}$, VCGs $2 \mathrm{~A}$ and $4 \mathrm{~B}$, and VCG 4A, and the existence of other VCGs or VCG subgroups that have been found rarely and seem to be genetically different: VCG 2B (2B-I, 2B-II, and 2B-III), VCG 3, and VCG 6. This is consistent with a population structure consisting of some recombination events (maybe extant, rare, or past), with a clonal expansion of certain successful individuals possibly associated with agriculture. It is possible that these latter groups have emerged through the evolutionary mechanisms described above but the one question arising is: where are these variants originating from? We believe that there are aspects of $V$. dahliae biology that have not yet been explored appropriately, and it is possible that there is a more complex diversity in $V$. dahliae that has not been targeted. For example, the diversity of soil $V$. dahliae populations has not been properly studied, and the fungus has been found in endophytic associations with asymptomatic hosts (34). Bell (6) suggested that the early VCG work done by Puhalla (44) using melanin-deficient mutants probably overestimated VCG diversity because later studies based on nit mutant assays lumped the 16 early VCGs into four VCGs $(26,27,51,52)$. However, the increasing use of molecular tools is resolving more finely the diversity in $V$. dahliae populations and identifying complex associations between VCGs. Although we are closer to understanding these relationships and the impact they have on $V$. dahliae biology, there is a clear need for more powerful molecular markers used in an ecologically broad context to resolve the origin and evolution of diversity in $V$. dahliae and its impact on the biology of this important fungus.

\section{ACKNOWLEDGMENTS}

This research was partially funded by the Sarah Chinn Kalser Faculty Research Assistance Endowment, College of Agricultural Sciences, The Pennsylvania State University. We thank all suppliers of $V$. dahliae isolates; J. Yanez, S. Colihan, C. Barrett, C. Black, and C. Olivares-García for excellent technical support; and D. Geiser for helpful discussions during the preparation of this manuscript.

\section{LITERATURE CITED}

1. Apple, D., and Gordon, T. R. 1996. Relationships among pathogenic and nonpathogenic isolates of Fusarium oxysporum based on the partial sequence of the intergenic spacer region of the ribosomal DNA. Mol. Plant-Microbe Interact. 9:125-138.

2. Atallah, Z. K., Maruthachalam K., du Toit, L., Koike, S. T., Klosterman, S. J., Hayes, R. J., and Subbarao, K. V. 2010. Population analyses of the vascular plant pathogen Verticillium dahliae detect recombination and transcontinental gene flow. Fungal Genet. Biol. 47:416-422.

3. Baayen, R. P., O’Donnell, K., Bonants, P. J. M., Cigelnik, E., Kroon, L. P. N. M., Roebroeck, E. J. A., and Waalwijk, C. 2000. Gene genealogies and AFLP analyses in the Fusarium oxysporum complex identify monophyletic and nonmonophyletic formae speciales causing wilt and rot disease. Phytopathology 90:891-900.

4. Bejarano-Alcázar, J., Blanco-López, M. A., Melero-Vara, J. M., and Jiménez-Díaz, R. M. 1996. Etiology, importance, and distribution of Verticillium wilt of cotton in southern Spain. Plant Dis. 80:1233-1238.

5. Bejarano-Alcázar, J., Melero-Vara, J. M., Blanco-López, M. A., and Jiménez-Díaz, R. M. 1995. Influence of inoculum density of defoliating and non-defoliating pathotypes of Verticillium dahliae on epidemics of Verticillium wilt of cotton in southern Spain. Phytopathology 85:14741481.

6. Bell, A. A. 1994. Mechanisms of disease resistance in Gossypium species and variation in Verticillium dahliae. Pages 225-235 in: Proc. World Cotton Res. Conf. G. A. Constable and N. W. Forrester, eds. CSIRO, Melbourne, Australia.

7. Berbegal, M., Garzón, C., Ortega, A., Armengol, J., Jiménez-Díaz, R. M., 
and Jiménez-Gasco, M. M. 2011. Development and application of new molecular markers for analysis of genetic diversity in Verticillium dahliae populations. Plant Pathol. 60:866-877.

8. Berbegal, M., Ortega, A., Jiménez-Gasco, M. M., Olivares-García, C., Jiménez-Díaz, R. M., and Armengol, J. 2010. Genetic diversity and host range of Verticillium dahliae isolates from artichoke and other vegetable crops in Spain. Plant Dis. 94:396-404.

9. Bhat, R. G., Smith, R. F., Koike, S. T., Wu, B. M., and Subbarao, K. V. 2003. Characterization of Verticillium dahliae isolates and wilt epidemics of pepper. Plant Dis. 87:789-797.

10. Cirulli, M., Bubici, G., Amenduni, M., Armengol, J., Berbegal, M., Jiménez-Gasco, M. M., and Jiménez-Diaz, R. M. 2010. Verticillium wilt: a threat to artichoke production. Plant Dis. 94:1176-1187.

11. Collado-Romero, M., Mercado-Blanco, J., and Jiménez-Díaz, R. M. 2010. DNA sequence analysis of conserved genes reveals hybridization events that increase genetic diversity in Verticillium dahliae. Fungal Biol. 114:209-218

12. Collado-Romero, M., Mercado-Blanco, J., Olivares-García, C., and Jiménez-Díaz, R. M. 2008. Phylogenetic analysis of Verticillium dahliae vegetative compatibility groups. Phytopathology 98:1019-1028.

13. Collado-Romero, M., Mercado-Blanco, J., Olivares-García, C., ValverdeCorredor, A., and Jiménez-Díaz, R. M. 2006. Molecular variability within and among Verticillium dahliae vegetative compatibility groups determined by fluorescent amplified fragment length polymorphism and polymerase chain reaction markers. Phytopathology 96:485-495.

14. Collins, A., Mercado-Blanco, J., Jiménez-Díaz, R. M., Olivares, C., Clewes, E., and Barbara, D. J. 2005. Correlation of molecular markers and biological properties in Verticillium dahliae and the possible origins of some isolates. Plant Pathol. 54:549-557.

15. Cooke, D. E. L., and Lees, A. K. 2004. Markers, old and new, for examining Phytophthora infestans diversity. Plant Pathol. 53:692-704.

16. Demirci, E., and Genc, T. 2009. Vegetative compatibility groups of Verticillium dahliae isolates from weeds in potato fields. J. Plant Pathol. 91:671-676.

17. Deprés, L., Gielly, L., Redoutet, B., and Tabarlet, P. 2003. Using AFLP to resolve phylogenetic relationships in a morphologically diversified plant species complex when nuclear and chloroplast sequences fail to reveal variability. Mol. Phylogenet. Evol. 27:185-196.

18. Dung, J. K. S., Peever, T. L., and Johnson D. A. 2013. Verticillium dahliae populations from mint and potato are genetically divergent with predominant haplotypes. Phytopathology 103:445-459.

19. Fourie, G., Steenkamp, E. T., Gordon, T. R., and Viljoen, A. 2009. Evolutionary Relationships among the Fusarium oxysporum f. sp. cubense vegetative compatibility groups. Appl. Environ. Microbiol. 74:4770-4781.

20. Glass, N. L., and Kaneko, I. 2003. Fatal attraction: nonself recognition and heterokaryon incompatibility in filamentous fungi. Eukaryot. Cell 2:1-8.

21. Glass, N. L., and Kuldau, G. A. 1992. Mating type and vegetative incompatibility in filamentous ascomycetes. Annu. Rev. Phytopathol. 30:201224.

22. Jiménez-Díaz, R. M. Cirulli, M., Bubici, G., Jiménez-Gasco, M. M., Antoniou, P. P., and Tjamos, E. C. 2012. Verticillium wilt: a major threat to olive production: current status and future prospects for its management. Plant Dis. 96:304-329.

23. Jiménez-Díaz, R. M., Mercado-Blanco, J., Olivares-García, C., ColladoRomero, M., Bejarano-Alcázar, J., Rodríguez-Jurado, D., Giménez-Jaime, A., García-Jiménez, J., and Armengol, J. 2006. Genetic and virulence diversity in Verticillium dahliae populations infecting artichoke in eastern-central Spain. Phytopathology 96:288-298.

24. Jiménez-Díaz, R. M., Olivares-García, C., Landa, B. B., Jiménez-Gasco, M. M., and Navas-Cortés, J. A. 2011. Region-Wide Analysis of genetic diversity in Verticillium dahliae populations infecting olive in southern Spain and agricultural factors influencing the distribution and prevalence of vegetative compatibility groups and pathotypes. Phytopathology 101:304-315.

25. Jiménez-Gasco, M. M., Milgroom, M. G., and Jiménez-Díaz, R. M. 2002. Gene genealogies support Fusarium oxysporum f. sp. ciceris as a monophyletic group. Plant Pathol. 51:72-77

26. Joaquim, T. R., and Rowe, R. C. 1990. Reassessment of vegetative compatibility relationships among strains of Verticillium dahliae using nitratenonutilizing mutants. Phytopathology 80:1160-1166.

27. Joaquim, T. R., and Rowe, R. C. 1991. Vegetative compatibility and virulence strains of Verticillium dahliae from soil and potato plant. Phytopathology 81:552-558.

28. Katan, T. 2000. Vegetative compatibility in populations of Verticilliuman overview. Pages 69-86 in: Advances in Verticillium: Research and Disease Management. E. C. Tjamos, R. C. Rowe, J. B. Heale, and R. D. Fravel, eds. American Phytopathological Society Press, St. Paul, MN.

29. Klosterman, S. J., Atallah, Z. K., Vallad, G. E., and Subbarao, K. V. 2009. Diversity, pathogenicity, and management of Verticillium species. Annu.
Rev. Phytopathol. 47:39-62.

30. Korolev, N., Pérez-Artés, E., Bejarano-Alcázar, J., Rodríguez-Jurado, D., Katan, J., Katan, T., and Jiménez-Díaz, R. M. 2001. Comparative study of genetic diversity and pathogenicity among populations of Verticillium dahliae from cotton in Spain and Israel. Eur. J. Plant Pathol. 107:443-456.

31. Leslie, J. F. 1993. Fungal vegetative compatibility. Annu. Rev. Phytopathol. 31:127-150.

32. López-Escudero, F. J., del Río, C., Caballero, J. M., and Blanco-López, M. A. 2004. Evaluation of olive cultivars for resistance to Verticillium dahliae. Eur. J. Plant Pathol. 110:79-85.

33. Ma, L. J., van der Does, H. C., Borkovich, K. A., Coleman, J. J., Daboussi, M. J., Di Pietro, A., Dufresne, M., Freitag, M., Grabherr, M., Henrissat, B., Houterman, P. M., Kang, S., Shim, W. B., Woloshuk, C., Xie, X., Xu, J. R., Antoniw, J., Baker, S. E., Bluhm, B. H., Breakspear, A., Brown, D. W., Butchko, R. A., Chapman, S., Coulson, R., Coutinho, P. M., Danchin, E. G., Diener, A., Gale, L. R., Gardiner, D. M., Goff, S., Hammond-Kosack, K, E., Hilburn, K., Hua-Van, A., Jonkers, W., Kazan, K., Kodira, C. D., Koehrsen, M., Kumar, L., Lee, Y. H., Li, L., Manners, J. M., Miranda-Saavedra, D., Mukherjee, M., Park, G., Park, J., Park, S. Y., Proctor, R. H., Regev, A., Ruiz-Roldan, M. C., Sain, D., Sakthikumar, S., Sykes, S., Schwartz, D. C., Turgeon, B. G., Wapinski, I., Yoder, O., Young, S., Zeng, Q., Zhou, S., Galagan, J., Cuomo, C. A., Kistler, H. C., and Rep, M. 2012. Comparative genomics reveals mobile pathogenicity chromosomes in Fusarium. Nature 464:367-373.

34. Malcolm, G. M., Kuldau, G. A., Gugino, B. K., and Jiménez-Gasco, M. M. 2013. Hidden host plant associations of soilborne fungal pathogens: an ecological perspective. Phytopathology 103:538-544.

35. Martin, F. N. 2010. Mitochondrial haplotype analysis as a tool for differentiating isolates of Verticillium dahliae. Phytopathology 100:12311239.

36. McAlpin, C. F., Horn, B. W., and Wicklow, D. T. 2005. DNA fingerprinting analysis of vegetative compatibility groups in Aspergillus caelatus. Mycologia 97:70-76.

37. Mercado-Blanco, J., Collado-Romero, M., Parrilla-Araujo, S., RodríguezJurado, D., and Jiménez-Díaz, R. M. 2003. Quantitative monitoring of colonization of olive genotypes by Verticillium dahliae pathotypes with real-time polymerase chain reaction. Physiol. Mol. Plant Pathol. 63:91105.

38. Milgroom, M. G., and Cortesi, P. 1999. Analysis of population structure of the chestnut blight fungus based on vegetative incompatibility genotypes. Proc. Natl. Acad. Sci. USA 96:10518-10523.

39. Nixon, K. C. 1999. The parsimony ratchet, a new method for rapid parsimony analysis. Cladistics 15:407-414.

40. O’Donnell, K., Gueidan, C., Sink, S., Johnston, P. R., Crous, P. W., Glenn, A., Riley, R., Zitomer, N. C., Colyer, P., Waalwijk, C., Lee, T. V., Moretti, A., Kang, S., Kim, H. S., Geiser, D. M., Juba, J. H., Baayen, R. P., Cromey, M. G., Bithell, S., Sutton, D. A., Skovgaard, K., Ploetz, R., Kistler, H. C., Elliott, M., Davis, M., and Sarver, B. A. 2009. A two-locus DNA sequence database for typing plant and human pathogens within the Fusarium oxysporum species complex. Fungal Genet. Biol. 46:936-948.

41. Omer, M. A., Johnson, D. A., Douhan, L. I., Hamm, P. B., and Rowe, R. C. 2008. Detection, quantification, and vegetative compatibility of Verticillium dahliae in potato and mint production soils in the Columbia Basin of Oregon and Washington. Plant Dis. 92:1127-1131.

42. Pegg, G. F., and Brady, B. L. 2002. Verticillium Wilts. CAB International, Wallingford, UK.

43. Pramateftaki, P. V., Antoniou, P. P., and Typas, M. A. 2000. The complete DNA sequence of the nuclear ribosomal RNA gene complex of Verticillium dahliae: intraspecific heterogeneity within the intergenic spacer region. Fungal Genet. Biol. 29:19-27.

44. Puhalla, J. E. 1979. Classification of Isolates of Verticillium dahliae based on heterokaryon Incompatibility. Phytopathology 69:1186-1189.

45. Puhalla, J. E., and Hummel, M. 1983. Vegetative compatibility groups within Verticillium dahliae. Phytopathology 73:1305-1308.

46. Qin, Q.-M., Vallad, G. E., Wu, B. M., and Subbarao, K. V. 2006. Phylogenetic analyses of phytopathogenic isolates of Verticillium spp. Phytopathology 96:582-592.

47. Remesal, E., Landa, B. B., Jiménez-Gasco, M. M., and Navas-Cortés, J. A. 2013. Sequence variation in two protein-coding genes correlates with mycelial compatibility groupings in Sclerotium rolfsii. Phytopathology 103:479-487.

48. Rowe, R. C., and Powelson, M. L. 2002. Potato early dying: management challenges in a changing production environment. Plant Dis. 86:11841193.

49. Shimodaira, H., and Hasegawa, M. 1999. Multiple comparisons of loglikelihoods with applications to phylogenetic inference. Mol. Biol. Evol. 16:1114-1116.

50. Sikes, D. S., and Lewis, P. O. 2001. Beta Software, Version 1. PAUPRat: PAUP* Implementation of the Parsimony Ratchet. Distributed by the authors. Department of Ecology and Evolutionary Biology, University of 
Connecticut, Storrs.

51. Strausbaugh, C. A. 1993. Assessment of vegetative compatibility and virulence of Verticillium dahliae isolates from Idaho potatoes and tester strains. Phytopathology 83:1253-1258

52. Strausbaugh, C. A., Schroth, M. N., Weinhold, A. R., and Hancock, J. G. 1992. Assessment of vegetative compatibility of Verticillium dahliae tester strains and isolates from California potatoes. Phytopathology 82:6168 .

53. Swofford, D. L. 2002. PAUP*. Phylogenetic Analysis using Parsimony (*and other methods), Version 4.0b10. Sinauer Associates, Sunderland, MA.

54. Taylor, J. W., Jacobson, D. J., and Fisher, M. C. 1999. The evolution of asexual fungi: reproduction, speciation and classification. Annu. Rev. Phytopathol. 37:197-246.

55. Tsror (Lahkim), L., Hazanovsky, M., Mordechi-Lebiush, S., and Sivan, S. 2001. Aggressiveness of Verticillium dahliae isolates from different vegetative compatibility groups to potato and tomato. Plant. Pathol. 50:477-482.

56. Typas, M. A. 1983. Heterokaryon incompatibility and interspecific hybridization between Verticillium albo-atrum and Verticillium dahliae following protoplast fusion and microinjection. J. Gen. Microbiol. 129:3043-3056.

57. Usami, T., Itoh, M., and Amemiya, Y. 2009. Asexual fungus Verticillium dahliae is potentially heterothallic. J. Gen. Plant Pathol. 75:422-427. 